1 Universidade Federal do Pará (UFPA), Instituto de Ciências da Saúde, Programa de PósGraduação em Saúde, Ambiente e Sociedade na Amazônia - Belém (PA), Brasil.

fabiogianb@gmail.com

2 Universidade Federal do Pará (UFPA), Instituto de Ciências da Saúde, Programa de Pós-

Graduação em Saúde, Ambiente e Sociedade na Amazônia - Belém (PA),

Brasil.

marcossilva@ufpa.br

3 Universidade Federal do Pará (UFPA), Instituto de Ciências da Saúde, Programa de PósGraduação em Saúde, Ambiente e Sociedade na Amazônia - Belém (PA),

Brasil.

marcieni@ufpa.br

4 Universidade Federal do Pará (UFPA), Museu Paraense Emílio Goeldi (MPEG) - Belém (PA),

Brasil.

alex@tabela.bio.br

\section{Avaliação do burnout em trabalhadores de um hospital universitário do município de Belém (PA)}

\author{
Evaluation of burnout in workers of a university hospital in the city of \\ Belém (PA)
}

Fábio Gian Braga Pantoja', Marcos Valério Santos da Silva², Marcieni Ataide de Andrade², Alex de Assis Santos dos Santos ${ }^{4}$

RESUMO Objetivou-se avaliar os elementos que caracterizam o burnout em trabalhadores de um hospital universitário do município de Belém, estado do Pará, Brasil. Trata-se de estudo descritivo, de abordagem quantitativa e de delineamento transversal, investigando-se 62 trabalhadores. Verificou-se alto índice de prevalência. Embora alguns resultados não confirmem a literatura, deve-se ressaltar que a maioria das categorias profissionais ainda não havia sido estudada adotando-se essa metodologia, o que impede comparações mais consistentes. Sugere-se que as condições de trabalho podem ser mais responsáveis pelos sintomas do burnout do que as características isoladas dos profissionais.

PALAVRAS-CHAVE Esgotamento profissional. Pessoal de saúde. Saúde do trabalhador.

\begin{abstract}
The objective of this study was to evaluate the elements that characterize burnout in workers at a university hospital in the city of Belém, state of Pará, Brazil. It is a descriptive study, with a quantitative approach and a cross-sectional delineation, investigating 62 workers. It was verified a high prevalence rate. Although some results do not confirm the literature, it must be emphasized that most of the professional categories had not yet been studied adopting this methodology, which prevents more consistent comparisons. It is suggested that working conditions may be more responsible for the symptoms of burnout rather than the isolated characteristics of the professionals.
\end{abstract}

KEYWORDS Burnout professional. Health personnel. Occupational health. 


\section{Introdução}

O trabalho na saúde se caracteriza pela sua relação com o outro, sendo que a particularidade desse tipo de trabalho justifica como ele vem sendo nomeado: trabalho afetivo ou prestação de cuidados (BERNARDES; PELLICCIOLI; GUARESCHI, 2010).

O trabalho afetivo é visível entre os que exercem o cuidado através da escuta e do acolhimento, como os enfermeiros, assistentes sociais, médicos, psicólogos, nutricionistas, entre outros, demonstrando que quando a produção afetiva se torna parte do trabalho assalariado, pode gerar alienação extrema, pois corre o risco de não ser compreendido como trabalho pelo fato de produzir afeto, conhecimentos e simbolismos (SODRÉ, 2013). Para Telles e Pimenta (2009), a atitude de ser solidário e de prestação de ajuda às pessoas é reconhecida como uma ação valorosa, mas não se pode desconsiderar que tal ação tem os seus custos emocionais.

O trabalho afetivo envolve, além da relação com o outro, a capacidade de produzir laços, redes sociais, invenções de novos desejos, exigindo do trabalhador da saúde sua inteligência, sensibilidade, imaginação, criatividade, conectividade e afetividade, ou seja, é a existência que é colocada a trabalhar, e não somente o corpo (BERNARDES; PELLICCIOLl; GUARESCHI, 2010).

Assim, existem diversas patologias que podem acometer os trabalhadores, como é possível verificar na lista de doenças relacionadas ao trabalho (BRASIL, 1999), na qual existem 198 entidades nosológicas classificadas, sendo uma delas o burnout, também conhecido como síndrome do esgotamento profissional.

O burnout é um tipo de resposta prolongada aos estressores emocionais e interpessoais crônicos no trabalho e recebe o código Z73.0 na classificação oficial. Envolve atitudes e condutas negativas com relação aos usuários, clientes, à organização e ao trabalho, em uma experiência subjetiva que ocasiona prejuízos práticos e emocionais para o trabalhador e a organização (BRASIL; OPAS/BRASIL, 2001).

Possui três elementos centrais: Exaustão Emocional (EE), que envolve sentimentos de desgaste emocional e esvaziamento afetivo; despersonalização (DP), onde se verifica reação negativa, insensibilidade ou afastamento excessivo do público que deveria receber os serviços ou cuidados; e diminuição do envolvimento pessoal no trabalho (RP), que envolve sentimentos de diminuição de competência e de sucesso no trabalho (BRASIL; OPAS/BRASIL, 2001).

Diversos estudos vêm sendo realizados, a fim de investigar o burnout em médicos, enfermeiros e educadores. Carece-se, entretanto, de pesquisas cuja população estudada seja composta por trabalhadores da área da saúde de outras categorias profissionais, cujo trabalho se caracterize como de natureza afetiva ou de cuidados, como os: assistentes sociais, farmacêuticos, fisioterapeutas, fonoaudiólogos, nutricionistas, odontólogos, psicólogos e terapeutas ocupacionais.

Diante desse contexto, este estudo investigou quais são os elementos que caracterizam o burnout em trabalhadores de um hospital universitário do município de Belém (PA), sendo que, para isso, também se investigaram as características demográfica, social, ocupacional e cultural desses trabalhadores, os níveis de burnout entre os mesmos e as associações entre tais variáveis e essa síndrome.

\section{Material e métodos}

A pesquisa se caracterizou como um estudo do tipo descritivo, através de abordagem quantitativa de delineamento transversal.

Foi realizada em um hospital universitário no município de Belém, capital do estado do Pará. Trata-se de uma instituição de assistência, ensino e pesquisa ligada à Universidade Federal do Pará (UFPA), que presta serviços à comunidade exclusivamente através do 
Sistema Único de Saúde (SUS). Possui programas de residência médica e multiprofissional, sendo referência na região Norte do Brasil em diversas áreas.

Os participantes da pesquisa foram os trabalhadores de nível superior da área da saúde que prestavam serviços no hospital, que possuíam vínculo formal de emprego junto à instituição, em atividade profissional há mais de um ano e cujas atividades fossem diretamente associadas aos cuidados com pacientes.

Foram excluídos da amostra aqueles que estavam em gozo de licença médica para tratamento de saúde. Justifica-se tal escolha considerando que os sujeitos que compõem a amostra possam estar enfrentando potenciais riscos de adoecimento, em detrimento dos trabalhadores já adoecidos.

Também foram excluídos os profissionais que estavam de férias; que haviam sido transferidos para outros órgãos; de licença maternidade ou afastados para capacitação profissional, como em cursos de mestrado ou doutorado, no momento em que a equipe se deslocou ao local de trabalho para coleta de dados.

Excluíram-se, ainda, aqueles trabalhadores que, embora constassem na listagem, haviam sido demitidos durante a fase da coleta de dados.

Considerando que, devido à frequência da incidência do burnout em médicos e enfermeiros já ter sido abordada em estudos anteriores, e por tratar-se de uma síndrome, portanto, relativamente conhecida nessas duas categorias profissionais, optou-se por não incluí-las nessa amostra, restando, assim, oito categorias profissionais que usualmente prestam serviços de saúde em ambientes hospitalares: assistentes sociais, farmacêuticos, fisioterapeutas, fonoaudiólogos, nutricionistas, odontólogos, psicólogos e terapeutas ocupacionais.

Foi fornecida a listagem nominal desses empregados pela direção do hospital, sendo convidados todos os trabalhadores que preenchiam os critérios de inclusão e que não se enquadravam nos critérios de exclusão.

A coleta de dados foi realizada durante os turnos de trabalho dos empregados por um período aproximado de 30 dias, entre os meses de novembro e dezembro de 2015 , sendo recrutados alunos de graduação da área da saúde que receberam treinamento para aplicação dos formulários e que atuaram como auxiliares na coleta.

Foram aplicados 2 (dois) questionários: 0 primeiro foi um protocolo que continha perguntas que possibilitassem a caracterização demográfica, social, ocupacional e cultural dos sujeitos, de elaboração do pesquisador.

$\mathrm{O}$ segundo questionário foi o Maslach Burnout Inventory - Human Services Survey (MBI-HSS). Utilizou-se a versão proposta por Carlotto e Câmara (2007), que analisaram as propriedades psicométricas desse instrumento quanto às suas fidedignidade $\mathrm{e}$ validade, em uma amostra multifuncional de trabalhadores brasileiros das áreas da saúde, justiça, segurança e educação, e nos informa que há consistência interna do MBI-HSS nas suas três dimensões na amostra estudada, demonstrando a capacidade desse inventário de medição do burnout em diversas profissões, sendo que a versão brasileira do instrumento apresenta os requisitos necessários em termos de consistência interna e validade fatorial para ser amplamente utilizada na avaliação da síndrome em trabalhadores que desenvolvem suas atividades em profissões consideradas de ajuda.

O MBI-HSS é composto por 22 afirmações sobre sentimentos e atitudes que englobam os três aspectos fundamentais do burnout, divididos em três escalas, com sete opções de respostas, do tipo Likert, variando de 0 a 6 . A EE é avaliada por nove itens, DP por cinco e RP por oito.

Realizou-se um teste piloto com 8 trabalhadores de três categorias profissionais no próprio hospital, o que apontou para a necessidade de se modificarem algumas opções de resposta de alguns itens do primeiro 
questionário, para melhor refinamento das análises estatísticas. Serviu, também, para alterar a disposição dos questionários quando da apresentação aos participantes. Todos os sujeitos que responderam ao teste piloto não foram incluídos quando da seleção da amostra da pesquisa.

Não há consenso na literatura para pontos de corte na avaliação do burnout. Dessa forma, optou-se por utilizar os seguintes critérios: para EE, uma pontuação maior ou igual a 27 indica alto nível; de 19 a 26, nível moderado; e menor que 19, nível baixo. Para DP, pontuações iguais ou maiores que 10 indicam alto nível; de seis a nove, nível moderado; e menores que seis, nível baixo. A pontuação relacionada à RP vai em direção oposta às outras, uma vez que pontuações de zero a 33 indicam alto nível; de 34 a 39, nível moderado; e maior ou igual a 40 , baixo. Tais pontos de corte foram utilizados em estudos com profissionais de saúde nas pesquisas de Tucunduva et al. (2006); Moreira et al. (2009); Mota, Dosea e Nunes (2014), entre outros.

Como existem estudos que consideram o burnout com a presença de apenas uma das dimensões do questionário Maslach em nível alto, bem como outros que avaliam a síndrome apenas se as três dimensões estiverem em níveis críticos (TUCUNDUVA ET AL., 2006; MOREIRA ET AL., 2009), avaliaram-se, neste estudo, as três dimensões do MBI, destacando-se graus médio e alto em cada dimensão, considerando a presença de burnout entre aqueles que apresentaram tanto uma quanto as três dimensões críticas.

Para análise dos dados, foi utilizada estatística descritiva e inferencial. A apresentação dos dados foi realizada por meio de distribuições de frequências absolutas e relativas. $\mathrm{O}$ teste do Qui-quadrado foi utilizado para avaliar as variáveis qualitativas. As variáveis quantitativas foram comparadas pelo teste de Kruskal-Wallis com pós-teste de Dunn, visto que não foram compatíveis com o padrão de normalidade, o qual foi avaliado pelo teste de D’Agostino-Pearson (AYRES ET AL., 2007).
Considerou-se nível de significância (nível alfa) de 5\%, sendo que as análises foram realizadas através dos softwares SAM (Statistical Analysis Model), versão 1.0, e BioEstat (versão 5.3, Instituto de Desenvolvimento Sustentável Mamirauá, Belém, Pará, Brasil).

O projeto deste estudo foi aprovado no Comitê de Ética em Pesquisa (CEP) do hospital conforme CAAE: 43931115.5.3001.0017 e seguiu as determinações da Resolução ${ }^{\circ}$ 466/2012 do Conselho Nacional de Saúde (BRASIL, 2012). Todos os trabalhadores que concordaram em participar da pesquisa assinaram um Termo de Consentimento Livre e Esclarecido (TCLE).

\section{Resultados}

O número total dos trabalhadores de nível superior na área da saúde, de acordo com a listagem dos empregados por lotação, com algum vínculo com o hospital, foi de 145 funcionários: 35 farmacêuticos, 4 fonoaudiólogos, 20 nutricionistas, 4 terapeutas ocupacionais, 23 assistentes sociais, 23 fisioterapeutas, 22 odontólogos e 14 psicólogos. Além desses, também constavam no quadro de trabalhadores do hospital 223 médicos e 121 enfermeiros. A classe médica representa a maior categoria de trabalhadores da área da saúde de nível superior no hospital, totalizando $45,60 \%$ de todos os profissionais.

Durante a fase da coleta de dados, ocorreram demissões em alguns setores da instituição, sendo que, em alguns casos, como na fonoaudiologia, houve a demissão dos profissionais que constavam na listagem fornecida, o que impossibilitou a sua inclusão na pesquisa.

Também não foi possível o acesso aos odontólogos do nosocômio, pois, durante a fase estipulada para coleta de dados, foi informado que não havia trabalhadores no local dessa categoria profissional, em virtude de reformas no setor. 
Assim, dos 145 profissionais que constavam na listagem, aproximadamente 87 preenchiam os critérios de inclusão (vínculo de trabalho formal junto ao hospital; em atividade profissional há mais de um ano; e que suas atividades fossem diretamente associadas aos cuidados com pacientes), respeitando-se, também, os critérios de exclusão delimitados neste estudo. Restaram: 20 assistentes sociais, 18 farmacêuticos, 18 fisioterapeutas, 19 nutricionistas, 8 psicólogos e 4 terapeutas ocupacionais. Ao final, 62 formulários foram preenchidos pelos sujeitos que aceitaram participar do estudo, sendo: 14 assistentes sociais, 10 farmacêuticos, 10 fisioterapeutas, 17 nutricionistas, 7 psicólogos e 4 terapeutas ocupacionais.

Os trabalhadores com vínculo com o hospital estavam distribuídos entre a UFPA, Fundação de Amparo e Desenvolvimento da Pesquisa (Fadesp) e Secretaria Estadual de Saúde (Sespa).

Os trabalhadores com vínculo de trabalho com a UFPA incluem os servidores da administração direta, com seleção através de concurso público. Já os empregados com vínculo pela Fadesp estão abrangidos pelo regime da Consolidação das Leis do Trabalho (CLT). Os vínculos com a Sespa incluem servidores cedidos pelo órgão e que prestam seus serviços laborais no hospital, sendo o vínculo de trabalho do tipo estatutário. Outros vínculos que também foram identificados no hospital incluem servidores da Secretaria de Saúde do município de Belém (Sesma), Fundação Centro de Hemoterapia e Hematologia do Pará (Hemopa) e Ministério da Saúde.

Com relação ao questionário demográfico, social, ocupacional e cultural, a maioria dos profissionais pesquisados era do sexo feminino $(87,1 \%)$, na faixa etária entre 40 a 61 anos $(58,1 \%)$ e casados ou em união estável (59,7\%). Com relação à escolaridade, 53,2\% possuem pós-graduação do tipo Lato Sensu. $38,7 \%$ vivem com 5 a 9 salários mínimos; $50 \%$ possuem vínculo de trabalho com a UFPA; $35,5 \%$ trabalham no hospital entre 1 e 4 anos; 59,7\% mantêm outra atividade remunerada; e 87,1\% possuem jornada de trabalho de até 6 horas. 96,8\% procuram um profissional de saúde sempre que sente necessidade. $82,3 \%$ não pensaram em mudar de profissão; e 88,7 \% não sentem a profissão menos interessante do que quando começaram. 64,5\% têm filhos, 90,3\% residem em Belém e 72,6\% se deslocam para o trabalho de condução própria.

Os resultados do questionário demográfico, social, ocupacional e cultural dos trabalhadores estão descritos na tabela 1.

\begin{tabular}{|c|c|c|c|}
\hline & $n$ & $\%$ & $p$-valor \\
\hline Sexo & & & $<0.0001^{\star}$ \\
\hline Masculino & 8 & 12.9 & \\
\hline Feminino & 54 & 87.1 & \\
\hline Vínculo & & & $0.0205^{*}$ \\
\hline UFPA* $^{*}$ & 31 & 50.0 & \\
\hline Fadesp & 16 & 25.8 & \\
\hline Outro & 15 & 24.2 & \\
\hline Idade (anos) & & & 0.2530 \\
\hline 23 а 39 & 26 & 41.9 & \\
\hline 40 a 61 & 36 & 58.1 & \\
\hline
\end{tabular}


Tabela 1. (cont.)

\begin{tabular}{|c|c|c|c|}
\hline Escolaridade & & & $<0.0001^{\star}$ \\
\hline Graduação & 5 & 8.1 & \\
\hline Lato Sensu* & 33 & 53.2 & \\
\hline Strictu Sensu & 24 & 38.7 & \\
\hline Estado Civil & & & $<0.0001^{\star}$ \\
\hline Solteiro & 18 & 29.0 & \\
\hline Casado* & 37 & 59.7 & \\
\hline Viúvo & 3 & 4.8 & \\
\hline Divorciado & 4 & 6.5 & \\
\hline Tempo Serviço & & & 0.2685 \\
\hline 1 a 4 anos & 22 & 35.5 & \\
\hline 5 a 9 anos & 13 & 21.0 & \\
\hline 10 a 14 anos & 15 & 24.2 & \\
\hline 15 ou mais & 12 & 19.4 & \\
\hline Profissão & & & 0.0604 \\
\hline Assistente Social & 14 & 22.6 & \\
\hline Farmacêutico & 10 & 16.1 & \\
\hline Fisioterapeuta & 10 & 16.1 & \\
\hline Nutricionista & 17 & 27.4 & \\
\hline Psicólogo & 7 & 11.3 & \\
\hline T. Ocupacional & 4 & 6.5 & \\
\hline Renda (Salário Mínimo - SM) & & & 0.6366 \\
\hline Até 4 SM & 20 & 32.3 & \\
\hline 5 a 9 SM & 24 & 38.7 & \\
\hline $10 \mathrm{SM}$ ou mais & 18 & 29.0 & \\
\hline Jornada Diária & & & $<0.0001^{\star}$ \\
\hline $4 a 6 h^{\star}$ & 54 & 87.1 & \\
\hline 8 horas & 4 & 6.5 & \\
\hline 12 horas & 4 & 6.5 & \\
\hline Procura Profissional de Saúde & & & $<0.0001^{\star}$ \\
\hline Sim & 60 & 96.8 & \\
\hline Não & 2 & 3.2 & \\
\hline Tem Filhos & & & $0.0309^{\star}$ \\
\hline $\operatorname{sim}$ & 40 & 64.5 & \\
\hline Não & 22 & 35.5 & \\
\hline Desinteresse pela Profissão & & & $<0.0001^{\star}$ \\
\hline Sim & 7 & 11.3 & \\
\hline Não & 55 & 88.7 & \\
\hline
\end{tabular}


Tabela 1. (cont.)

\begin{tabular}{|c|c|c|c|}
\hline Pensou Mudar de Profissão & & & $<0.0001^{\star}$ \\
\hline $\operatorname{Sim}$ & 11 & 17.7 & \\
\hline Não & 51 & 82.3 & \\
\hline Reside em Belém & & & $<0.0001^{\star}$ \\
\hline Sim & 56 & 90.3 & \\
\hline Não & 6 & 9.7 & \\
\hline Condução Própria & & & $0.0006^{\star}$ \\
\hline Sim & 45 & 72.6 & \\
\hline Não & 17 & 27.4 & \\
\hline Outro Emprego & & & 0.1624 \\
\hline $\operatorname{Sim}$ & 37 & 59.7 & \\
\hline Não & 25 & 40.3 & \\
\hline
\end{tabular}

Fonte: Elaboração própria

*Qui-quadrado de aderência, com proporções esperadas iguais.

Os resultados do MBI mostram que, dos profissionais estudados, $48,3 \%$ apresentaram grau médio ou alto de exaustão emocional; 40,03\% médio ou alto de realização pessoal no trabalho; e 38,7\% apresentaram grau médio ou alto de despersonalização. De acordo com os dados desta pesquisa, $45,16 \%$ dos trabalhadores apresentaram pelo menos uma das dimensões do instrumento de Maslach em níveis críticos, indicando, de acordo com critérios que vêm sendo utilizados em alguns estudos, a presença do burnout, sendo que 8,06\% apresentaram todas as três dimensões críticas (tabela 2). Para identificação das dimensões do MBI entre os profissionais, elaborou-se a figura 1.

Tabela 2. Resultados do MBI entre os trabalhadores entrevistados

\begin{tabular}{lrrrrrr}
\hline & \multicolumn{2}{r}{ Exaustão Emocional } & \multicolumn{2}{r}{ Realização Pessoal } & \multicolumn{2}{r}{ Despersonalização } \\
\hline MBI & $\mathbf{n}$ & $\%$ & $\mathbf{n}$ & $\%$ & $\mathbf{n}$ & $\%$ \\
\hline Alto & 19 & 30.6 & 10 & 16.1 & 17 & 27.4 \\
Médio & 11 & 17.7 & 15 & 24.2 & 7 & 11.3 \\
Baixo & 32 & 51.6 & 37 & 59.7 & 38 & 61.3 \\
Médio OU Alto & 30 & 48.3 & 25 & 40,3 & 24 & 38.7 \\
\hline
\end{tabular}

Fonte: Elaboração própria.

p-valor $=0.1711$, Qui-quadrado (comparando os 3 domínios). 
Figura 1. Distribuição das dimensões do MBI entre os trabalhadores entrevistados

$n=62$

Profissionais da Área da Saúde

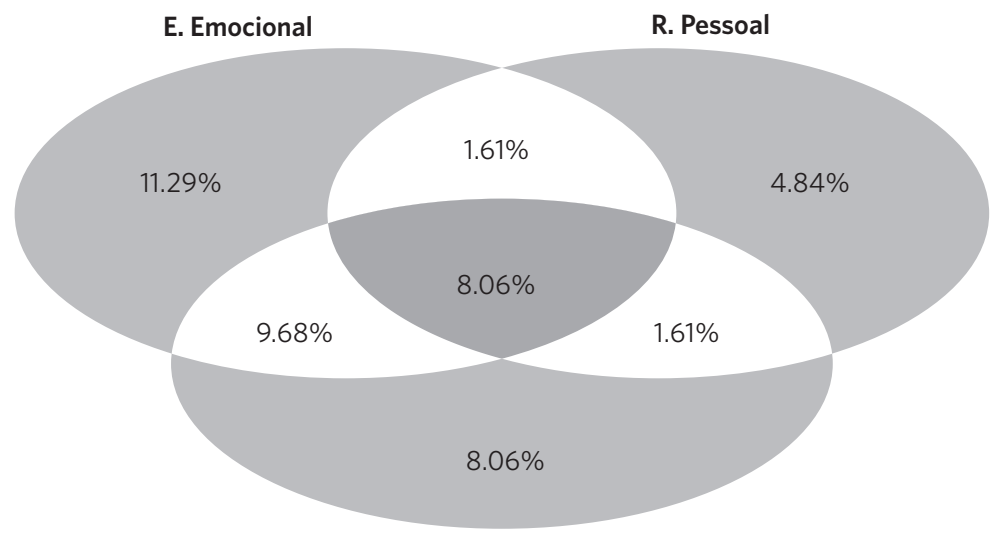

$54.84 \%$

Despersonalização

Fonte: Elaboração própria.

Considerando a associação entre as características dos profissionais assinaladas nos itens do questionário demográfico, social, ocupacional e cultural e os domínios do
MBI, observaram-se diferenças significativamente estatísticas nas variáveis: estado civil, renda, interesse pela profissão e outra atividade remunerada (tabela 3 ).

Tabela 3. Comparação estatística entre as diferentes dimensões do MBI e as características dos trabalhadores entrevistados

\begin{tabular}{lrrr}
\hline & Exaustão Emocional & Realização Pessoal & Despersonalização \\
\hline Sexo & 0.1859 & 0.1894 & 0.2314 \\
Vínculo & 0.2576 & 0.5144 & 0.3466 \\
Idade (anos) & 0.4582 & 0.9829 & 0.9205 \\
Escolaridade & 0.5458 & 0.6359 & 0.9639 \\
Estado Civil & $0.0285^{\star}$ & $0.0082^{\star}$ & 0.5263 \\
Tempo Serviço & 0.2659 & 0.8321 & 0.7791 \\
Profissão & 0.4099 & 0.8795 & 0.4641 \\
Renda (SM) & $0.0122^{\star}$ & 0.0636 & $0.0498^{\star}$ \\
Jornada Diária & 0.3041 & 0.5821 & 0.4501 \\
Proc. Prof. Saúde & $\mathrm{n} / \mathrm{a}$ & $\mathrm{n} / \mathrm{a}$ & $\mathrm{n} / \mathrm{a}^{\star *}$ \\
Tem Filhos & 0.0681 & 0.0617 & 0.1297 \\
Desint. Profissão & 0.0734 & $0.0011^{\star}$ & $0.0046^{\star}$ \\
P. Mudar Profissão & 0.5554 & 0.0883 & 0.1642 \\
Reside Belém & 0.7211 & 0.3848 & 0.3593 \\
Condução Própria & 0.6136 & 0.8113 & 0.9937 \\
Outro Emprego & $0.0373^{\star}$ & 0.6465 & 0.2755 \\
\hline Fone:Elaraça
\end{tabular}

Fonte: Elaboração própria.

*Kruskal-Wallis com pós-teste de Dunn.

${ }^{*}$ n/a: o teste de hipótese não foi aplicado. 
No tocante ao estado civil, observouse o burnout (pelo menos uma dimensão crítica) em: solteiro (13 casos, $72 \%$ ), casado/ união estável (12 casos, 32\%), divorciado ( 2 casos, 50\%), viúvo (1 caso, 33,3\%). Assim, solteiros apresentaram maior prevalência da síndrome.

Quanto à renda, observou-se a síndrome entre: aqueles que recebem até 4 salários mínimos (9 casos, 45\%), de 5 a 9 salários mínimos (5 casos, $21 \%$ ) e mais de 10 salários mínimos (14 casos, 78,6\%). Os dados mostram maior prevalência do burnout entre aqueles que recebem mais de 10 salários mínimos.

A prevalência da síndrome foi significativamente maior entre os trabalhadores que têm outra atividade remunerada (18 casos, 48,6\%), em comparação com aqueles que não têm (10 casos, 27\%).

Com relação ao item do questionário 'sente a profissão menos interessante do que quando começou', todos os que responderam 'sim' (7 casos, 100\%) apresentaram pelo menos uma dimensão crítica. Os restantes responderam negativamente (21 casos, 38\%).

A tabela 4 revela a distribuição do burnout entre os trabalhadores entrevistados, percebendo-se que alguns profissionais apresentaram mais os sintomas da síndrome do que outros, embora não se tenha encontrado diferença estatisticamente significativa.

Tabela 4. Distribuição do burnout por categoria profissional entre os trabalhadores entrevistados

\begin{tabular}{lrrrr} 
& \multicolumn{3}{c}{ Burnout } \\
\cline { 2 - 5 } & \multicolumn{2}{c}{ Presente } & \multicolumn{1}{c}{ Ausente } \\
\cline { 2 - 5 } & $\mathbf{n}$ & $\%$ & $\mathbf{n}$ & $\%$ \\
\hline Assistente Social & 7 & 50.0 & 7 & 50.0 \\
Farmacêutico & 5 & 50.0 & 5 & 50.0 \\
Fisioterapeuta & 2 & 20.0 & 8 & 80.0 \\
Nutricionista & 9 & 52.9 & 8 & 47.1 \\
Psicólogo & 3 & 42.9 & 4 & 57.1 \\
T. Ocupacional & 2 & 50.0 & 2 & 50.0 \\
Total geral & 28 & 45.2 & 34 & 54.8 \\
\hline
\end{tabular}

Fonte: Elaboração própria.

P-valor =0.6313, Qui-quadrado de independência, para comparar as diferenças conforme as profissões.

\section{Discussão}

A disparidade observada entre o quantitativo de profissionais da categoria médica, em detrimento das demais, embora não seja objetivo deste trabalho, suscita algumas questões de interesse da saúde coletiva.

O caso de uma equipe multiprofissional que não trabalhe com a interdisciplinaridade - compreendida como a utilização de técnicas metodológicas, conceitos e análises de diferentes ramos do saber com a finalidade de integração - pode caracterizar apenas burocratização, devido à mera comunicação de ideias, pois existem equipes multiprofissionais que apenas se ocupam de elaborar uma parte do trabalho, mesmo sem estarem imbuídas da preocupação com a integração de diferentes saberes. Nesse tipo de equipe, embora os profissionais pratiquem atividades distintas, vindo a ocupar o mesmo espaço físico e institucional, a decisão é, em 
geral, do médico, sendo levada a efeito por outros profissionais (BRUSCATO ET AL., 2004).

Não é possível afirmar que tal realidade ocorre na instituição pesquisada. Porém, cabe levantar essa discussão em virtude da distribuição observada na classe médica, cuja categoria representa quase metade dos profissionais da área da saúde de nível superior que trabalham no hospital, estando distribuídos entre diversas unidades do estabelecimento, incluindo clínicas, ambulatórios, salas de cirurgias e unidades de terapia intensiva.

Também não se pode afirmar que uma simples aproximação quantitativa no número de profissionais levaria a uma mudança de paradigma. Observa-se uma crise na medicina que envolve os planos ético, político, pedagógico e social. Entre os muitos problemas observados nesse campo está a competição entre as especialidades médicas e os demais profissionais de saúde, bem como a incapacidade de se formarem profissionais de saúde que sejam aptos à resolução dos problemas na área (GUEDES; NOGUEIRA; CAMARGO JÚNIOR, 2006).

No que se refere às demissões, entre o fornecimento da listagem pelo hospital e o início da coleta de dados, verificaram-se diversos desligamentos abrangendo os trabalhadores cujo vínculo de trabalho era do tipo temporário. Tal situação também fora divulgada pelo sindicato representante dos trabalhadores (MELO, 2015). Não foi possível saber se esses trabalhadores ficaram desempregados, pois tais sujeitos não compuseram a amostra, visto que o vínculo formal de trabalho era critério de inclusão dos participantes na pesquisa.

Acentua-se, entretanto, o impacto dessa realidade no mundo do trabalho. O desemprego, a informalidade e, sobretudo, a exclusão do mercado de trabalho estão associados a piores condições de saúde na população brasileira (GIATTI; BARRETO, 2006; MIQUILIN, 2013). É evidente, também, a sobrecarga de trabalho entre os que permanecem empregados, visto que, muitas vezes, não há reposição desses postos de trabalho.
Tal tipo de demissão produz insegurança e medo, que podem multiplicar os esforços do trabalhador, mas que também podem, ao contrário, reduzir a sua produtividade e/ou aumentar o número de acidentes e consequentes indenizações. Além do mais, não faz sentido submeter alguém às razões da economia, que é uma criação do ser humano e deveria lhe servir (VIANA, 2007).

Com relação à taxa de resposta de outros estudos que investigaram burnout entre profissionais de saúde, verifica-se que, no estudo de Tucunduva et al. (2006), a taxa de resposta obtida foi de $21,1 \%$, onde, de um total de 645 cartas enviadas, obteve-se um retorno de 136 formulários. Em outro estudo, Mota, Dosea e Nunes (2014) obtiveram $81,9 \%$ de taxa de resposta, pois foram respondidos 222 questionários de um total de 271 questionários da amostra, cuja totalidade dos servidores era de 894 sujeitos. Moreira et al. (2009) coletou $54,12 \%$ da amostra, uma vez que obteve 151 questionários de um total de 279 funcionários. O presente estudo obteve 62 participantes de um total de 87 , o que representa uma taxa de resposta de $71,26 \%$, estando, assim, de acordo com a média das taxas de resposta de outros estudos encontrados na literatura que investigaram burnout.

Os resultados do MBI demostram um percentual elevado de trabalhadores que apresentaram grau médio ou alto nas dimensões da síndrome, sendo que, conforme Codo e Vasques-Menezes (1999), um nível moderado de burnout já é preocupante do ponto de vista epidemiológico, devendo haver intervenção, pois o processo já se encontra em curso.

Analisando a saúde mental dos trabalhadores de um hospital, Ramminger (2002) concluiu que o estresse foi a queixa principal entre os funcionários, seguido da depressão, sustentando que, mesmo a literatura especializada não conseguindo definir com precisão o conceito de estresse, é unânime em afirmar sua relação com o trabalho.

Em um estudo realizado com médicos cancerologistas, $52,3 \%$ e $3 \%$ deles apresentaram, 
respectivamente, uma e as três dimensões consideradas críticas no questionário de Maslach (TUCUNDUVA ET AL., 2006). Os dados desta pesquisa estão de acordo quando considerada uma dimensão, mas divergem ao considerar as três dimensões, onde se encontraram percentis bem acima, com alterações em todas elas.

Outro estudo, com enfermeiros, técnicos e auxiliares de enfermagem, localizou $35,7 \%$ com pelo menos uma dimensão alterada, sendo que não houve trabalhadores com as três dimensões críticas (MOREIRA ET AL., 2009). Da mesa forma, os dados deste estudo se assemelham aos do estudo mencionado quando considerada apenas uma dimensão; e se diferem quando consideradas as três dimensões.

Quanto à análise do questionário demográfico, social, ocupacional e cultural, Gómez et al. (2005) afirmam que pessoas solteiras são mais propensas a apresentar a síndrome. Noro (2004), em seus estudos sobre trabalhadores hospitalares, evidenciou um aumento na síndrome entre os trabalhadores separados, embora sem diferença que possa ser considerada significativa, achados esses que corroboram os resultados desta pesquisa. Tais estudos sugerem que casados ou em união estável podem experimentar sentimentos de responsabilidade na família, além de apoio emocional proveniente dos familiares, podendo resultar em uma maior resistência ao burnout.

Com relação à renda, Albuquerque, Melo e Araújo Neto (2012) acreditam que grupos de trabalhadores que possuem salários menores apresentam maiores índices de burnout, mesmo que em seus achados não tenham percebido diferenças estatisticamente significativas entre os índices de profissionais de nível superior (que possuem maior renda) e os de níveis médio e técnico (que possuem menor renda), o que leva a crer que a sobrecarga de trabalho parece ser um fator de risco para a síndrome, visto que, entre os 14 casos identificados como burnout, entre os que recebem mais de 10 salários mínimos, existem $11(78,6 \%)$ que possuem outro emprego. A sobrecarga de trabalho tem sido uma das variáveis mais apontadas como predisponentes ao burnout (BENEVIDES-PEREIRA, 2008).

Nesse sentido, achados de Trindade et al. (2010), ao entrevistar trabalhadores portadores da síndrome, concluíram que o salário foi apontado como a segunda maior fonte de satisfação no trabalho para esse grupo, sendo que, para alguns desses trabalhadores, o salário foi apontado como a única fonte de satisfação. Tais autores acreditam que a tentativa de conciliar dois empregos é comum entre os trabalhadores da saúde, nos turnos da noite e do dia, o que pode afetar o comprometimento do profissional com seu trabalho.

Outra questão que merece atenção foram os resultados encontrados na variável outra atividade remunerada. Dados de outro estudo revelam que trabalhadores de outras categorias profissionais - diferentes das aqui pesquisadas - que possuem outro trabalho apresentaram menores níveis de burnout em comparação com os que não possuem (ALBUQUERQUE; MELO; ARAÚJO NETO, 2012).

Este estudo, que investigou somente profissionais de nível superior de escolaridade, encontrou resultado adverso, e tal diferença é atribuída, principalmente, a dois fatores: diferença de trabalho entre os profissionais, bem como devido à heterogeneidade entre os sujeitos pesquisados, visto que Albuquerque, Melo e Araújo Neto (2012) investigaram um conjunto de profissionais com níveis de exigência que abrangem desde o nível fundamental até o nível superior para o exercício de suas profissões. Já Mota, Dosea e Nunes (2014), investigando categoria profissional sem exigência de nível superior, não observou diferença estatística significativa.

Nesse sentido, ainda que o grau de escolaridade não seja um fator determinante para a síndrome, em seus achados, Albuquerque, Melo e Araújo Neto (2012) constataram, embora sem encontrar diferença significativa, que trabalhadores que possuem nível 
superior apresentam burnout mais desenvolvido quando comparados com aqueles que possuem somente nível técnico/médio.

O desinteresse pela profissão está relacionado com o próprio burnout, sendo a síndrome associada especificamente ao mundo laboral (BENEVIDES-PEREIRA, 2008). Pelas definições de Magalhães e Glina (2006), está no próprio conceito da síndrome, quando afirmam que o burnout ocorre quando o estresse crônico consome o trabalhador física e emocionalmente, deixando-o agressivo e irritadiço e/ou desmotivado, desinteressado, causando um mal-estar interno, insatisfação ocupacional e insensibilidade com relação a quase tudo e todos. Para Codo e VasquesMenezes (1999), o trabalhador perde o sentido da sua relação com o trabalho.

Tais constatações confirmam os dados deste estudo, no qual a totalidade dos trabalhadores que manifestaram desinteresse pela profissão apresenta pelo menos uma dimensão crítica.

Ainda que utilizados o mesmo instrumento e pontos de cortes semelhantes aos desta pesquisa, atribuem-se essas divergências às diferenças entre a população estudada, no que se refere à categoria profissional e/ou ao nível de escolaridade e/ou ao local de trabalho diferente.

É importante frisar a presença da síndrome entre os trabalhadores e suas respectivas categorias profissionais, pois, mesmo sem perceber diferenças estatisticamente significativas entre os mesmos, há necessidade de investigações mais profundas para que seja possível explicar os índices verificados.

Uma limitação deste estudo foi a não caracterização de cada profissional quanto à área específica de sua atuação, como, por exemplo, unidades de diagnóstico de meningite ou setores da pediatria. Entretanto, por se tratar de categorias profissionais que ainda não haviam sido investigadas, e dado o número reduzido de profissionais como um todo, acredita-se que este estudo reflete as características da síndrome nos profissionais que compuseram a amostra no universo do contexto hospitalar.

Outra limitação foram as impossibilidades de se abranger todas as categorias profissionais que haviam sido previstas no projeto. Entretanto, acredita-se que a amostra foi representativa, visto que se buscou a totalidade dos trabalhadores, além de que a taxa de resposta obtida está de acordo com a de outros estudos.

Importante destacar que não há na literatura um consenso para a definição da síndrome a partir dos valores encontrados no questionário Maslach. Grande parte dos estudos descreve os níveis obtidos nas três diferentes dimensões, entretanto, sem um critério definido para agrupá-las. Dessa forma, a definição do burnout se confunde com as próprias dimensões verificadas. Apesar de o questionário Maslach ser específico para o diagnóstico da síndrome, a falta de padronização para sua avaliação dificulta a interpretação dos resultados (TUCUNDUVA ET AL., 2006).

Por não haver consenso para a interpretação do MBI, critérios diferentes são utilizados em publicações com o intuito de classificar e diagnosticar burnout. Nesse sentido, a diversidade de estudos acadêmicos, no que se refere às formas de avaliação do burnout com relação aos pontos de cortes do instrumento para o estabelecimento de suas dimensões em níveis de prevalência alto, moderado e baixo, pode configurar um fator impeditivo para o estabelecimento de comparações mais consistentes com os resultados de outros trabalhos pesquisados (MOREIRA ET AL., 2009).

\section{Conclusões}

As constatações do presente estudo demonstram que o índice de prevalência encontrado entre o grupo de trabalhadores pesquisados pode ser considerado alto, uma vez que quase metade deles apresentou características 
compatíveis com a síndrome, o que impacta a saúde dos mesmos.

O estudo revela, ainda, que diversos trabalhadores da área da saúde estão sujeitos a ser afetados pelo burnout, visto que algumas categorias profissionais ainda não haviam sido investigadas segundo a metodologia ora utilizada com relação aos níveis da síndrome. Esse fato, porém, prejudica o estabelecimento de comparações mais rijas.

Como grande parte das associações estudadas não obteve significância estatística, é possível sugerir que as condições de trabalho podem ser as responsáveis pelos sintomas do burnout em maior proporção do que as características isoladas dos profissionais ou de seu ambiente de trabalho, sendo também essa a conclusão de Moreira et al. (2009). Acredita-se, assim, que as demissões observadas no estabelecimento durante a pesquisa estão entre os fatores que contribuíram para os níveis observados de burnout.

Diversos autores identificam ações que poderiam minimizar os efeitos da síndrome, como: criação de espaços coletivos dentro das instituições para o compartilhamento de sentimentos (MOTA; DOSEA; NUNES, 2014). Também, o fomento de valores pessoais e familiares, a fim de incentivar os trabalhadores a aproveitar os momentos de convivência com a família e com os companheiros de trabalho, além da programação de medidas que favoreçam o controle dos níveis de estresse dos trabalhadores, entre outras ações dessa natureza (TRINDADE ET AL., 2010).

Não se discorda dessas ações. Entretanto, o burnout é um problema do mundo do trabalho e de saúde pública que nasce a partir da exploração do trabalhador pelo capital. Dessa forma, sugere-se o engajamento e a militância política dos trabalhadores visando ao fortalecimento de suas entidades representativas de classe para que seja possível auferir conquistas coletivas, garantindo sua saúde tanto física quanto mental.

É importante a realização de novos estudos, sobretudo qualitativos, que busquem aprofundar a investigação dos fatores desencadeantes e ou associados ao burnout entre todos os trabalhadores. 


\section{Referências}

\section{ALBUQUERQUE, F. J. B.; MELO, C. F.; ARAÚJO}

NETO, J. L. Avaliação da Síndrome de Burnout em Profissionais da Estratégia Saúde da Família da Capital Paraibana. Psicologia: Reflexão e Crítica, Porto Alegre, v. 25, n. 3, p. 542-549, 2012. Disponível em: <http:// www.scielo.br/pdf/prc/v25n3/v25n3a14.pdf >. Acesso em: 19 jan. 2016.

AYRES, M.; AYRES JR., M.; AYRES, D. L.; SANTOS, A. A. S. BioEstat 5.3: Aplicações Estatísticas nas Áreas das Ciências Biológicas e Médicas. 5. ed. Belém: Publicações Avulsas do Mamirauá, 2007.

BENEVIDES-PEREIRA, A. M. T. Burnout, por quê?? In: BENEVIDES-PEREIRA, A. M. T. Burnout: quando o trabalho ameaça o bem-estar do trabalhador. São Paulo: Casa do Psicólogo, 2008. p. 13-20. Disponível em: $<$ https://books.google.com.br/books?hl=pt-BR\&lr=\&id=EMnnJklADqIC\&oi=fnd\&pg=PA9\& $\mathrm{dq}=$ burnout + desinteresse + profiss $\% \mathrm{C} 3 \% \mathrm{~A} 3 \mathrm{o} \&$ ots $=$ rcmAAUW1Jj\&sig=8S4fZM8VqcuK9uttL27nZoV 1S6I\#v=onepage \&q=burnout\%20desinteresse $\% 20$ profiss $\%$ C3\%A3o\&f=false>. Acesso em: 18 jan. 2016.

BERNARDES, A. G.; PELLICCIOLI, E. C.; GUARESCHI, N. M. F. Trabalho e produção de saúde: práticas de liberdade e formas de governamentalidade. Psicologia \&t Sociedade, Belo Horizonte, v. 22, n. 1, p. 5-13, 2010. Disponível em: <http://www.scielo.br/pdf/ psoc/v22nl/v22nla02.pdf >. Acesso em: 25 ago.2015.

BRASIL. Conselho Nacional de Saúde. Resolução $\mathrm{n}^{\circ} 466$, de 12 de dezembro de 2012. Diário Oficial [da] União, Brasília, DF, 12 dez. 2012. Disponível em: $<$ http://conselho.saude.gov.br/resolucoes/2012/ Reso466.pdf >. Acesso em: 22 dez. 2014.

Ministério da Saúde. Portaria n ${ }^{0}$ 1.339/GM, de 18 de novembro de 1999. Institui a Lista de Doenças relacionadas ao Trabalho. Diário Oficial [da] União, Brasília, DF, 12 nov. 1999. Disponível em: <http:// bvsms.saude.gov.br/bvs/saudelegis/gm/1999/ prt1339_18_11_1999.html>. Acesso em: 22 dez. 2014.

Ministério da Saúde; ORGANIZAÇÃO
PAN-AMERICANA DA SAÚDE NO BRASIL (OPAS/

BRASIL). Doenças relacionadas ao trabalho: manual de procedimentos para os serviços de saúde. Brasília, DF: Ministério da Saúde, 2001. (Série A. Normas e manuais técnicos; n. 114).

BRUSCATO, W. L. et al. O trabalho em equipe multiprofissional. In: BRUSCATO, W. L.; BENEDETTI, C.; LOPES, S. R. A. (Org.). A prática da psicologia hospitalar na Santa Casa de São Paulo: novos paradigmas em uma antiga história. São Paulo: Casa do Psicólogo, 2004. p. 33-42. Disponível em: <https://books.google. com.br/books?hl=pt-BR\&lr=\&id=plkploewiYOC\&oi $=$ fnd \&pg=PA33\&dq=equipe+multiprofissional + hosp ital\&ots=7qAepsQ8ms\&sig=Pzd-h5L41-1gePPv46K uZfvUDc\#v=onepage\&q=equipe\%20multiprofissional\%20hospital\&f=false>. Acesso em: 1 dez. 2015.

CARLOTTO, M. S.; CÂMARA, S. G. Propriedades psicométricas do Maslach Burnout Inventory em uma amostra multifuncional. Estudos de Psicologia, Campinas, v. 24, n. 3, p. 325-332, jul./set. 2007. Disponível em: <http://www.scielo.br/scielo. php?pid=S0103-166X2007000300004\&script=sci abstract\&tlng=pt>. Acesso em: 1 dez. 2015.

CODO, W.; VASQUES-MENEZES, I. O que é burnout. In: CODO, W. (Coord.). Educação: carinho e trabalho. Petrópolis: Vozes; Brasília, DF: Confederação Nacional dos Trabalhadores em Educação; Universidade de Brasília, 1999. p. 237-254.

GIATTI, L.; BARRETO, S. M. Situação do indivíduo no mercado de trabalho e iniqüidade em saúde no Brasil. Revista de Saúde Pública, São Paulo, v. 40, n. 1, p. 99-106, 2006. Disponível em: <http://www.scielo.br/pdf/rsp/ v40nl/27122.pdf>. Acesso em: 1 dez. 2015.

GOMÉZ, M. N. et al. Relación entre perfil psicológico, calidad de vida y estrés asistencial en personal de enfermería. Universitas Psychologica, Bogotá, v. 4, n.1, p. 63-75, jan./jun., 2005. Disponível em: <http://pepsic. bvsalud.org/pdf/up/v4nl/v4nla09.pdf >. Acesso em: 12 jan. 2016. 
GUEDES, C. R.; NOGUEIRA, M. I.; CAMARGO

JÚNIOR, K. R. A subjetividade como anomalia: contribuições epistemológicas para a crítica do modelo biomédico. Ciência \& Saúde Coletiva, Rio de Janeiro, v. 11, n. 4, p. 1093-1103, 2006. Disponível em: <http:// www.scielo.br/pdf/csc/v1ln4/32345.pdf>. Acesso em: 1 dez. 2015.

MAGALHÃES, R. A. C.; GLINA, D. M. R. Prevalência de Burnout em médicos de um Hospital Público de São Paulo. Saúde, Ética \&t Justiça, São Paulo, v. 11, n. 1/2, p. 29-35. 2006. Disponível em: <http://www.revistas.usp. br/sej/article/viewFile/43921/47542>. Acesso em: 18 jan. 2016.

MIQUILIN, I. O. C. et al. Desigualdades no acesso e uso dos serviços de saúde entre trabalhadores informais e desempregados: análise da PNAD 2008, Brasil. Cadernos de Saúde Pública, Rio de Janeiro, v. 29, n. 7, p. 1392-1406, jul. 2013. Disponível em: <http://www.scielosp.org/pdf/csp/ v29n7/13.pdf>. Acesso em: 1 dez. 2015.

MOREIRA, D. S. et al. Prevalência da síndrome de burnout em trabalhadores de enfermagem de um hospital de grande porte da Região Sul do Brasil. Cadernos de Saúde Pública, Rio de Janeiro, v. 25, n. 7, p. 1559-1568, jul. 2009. Disponível em: <http://www.scielo.br/pdf/ csp/v25n7/14.pdf>. Acesso em: 20 dez. 2014.

MOTA, C. M.; DOSEA, G. S.; NUNES, P. S. Avaliação da presença da Síndrome de Burnout em Agentes Comunitários de Saúde no município de Aracaju, Sergipe, Brasil. Ciência \& Saúde Coletiva, Rio de Janeiro, v. 19, n. 12, dez. 2014.

NORO, N. T. T. Síndrome de burnout entre trabalhadores de um hospital geral. 2004. 74 f. Dissertação (Mestrado em Engenharia de Produção com ênfase em ergonomia), Universidade Federal do Rio Grande do Sul, Porto Alegre, 2004.

RAMMINGER, T. A saúde mental do trabalhador em saúde mental: um estudo com trabalhadores de um hospital psiquiátrico. Boletim da Saúde, Porto Alegre, v. 16, n. 1, 2002. p. 111-124. Disponível em: <http://bvsms. saude.gov.br/bvs/periodicos/boletim_saude_v16nl. pdf\#page=107>. Acesso em: 3 dez. 2015.
MELO, F. Trabalhadores do Hospital Barros Barreto denunciarão demissões e precarização em audiência pública. SINDIIFES, Belém, 14 jul. 2015. Disponível em: <http://www.sindtifes.org.br/index. php?option=com $\_$content $\&$ view $=$ article $\&$ id $=1044$ : trabalhadores-do-hospital-barros-barreto-denunciarao-demissoes-e-precarizacao-em-audiencia-publica\&catid=1:latest-news\&Itemid=49>. Acesso em: 13 nov. 2015.

SODRÉ, F. O trabalho e as redes. In: MINAYO GOMEZ, C.; MACHADO, J. M. H.; PENA, P. G. L. Saúde do trabalhador na sociedade brasileira contemporânea. Rio de Janeiro: Fiocruz, 2013, p. 297-314.

TELLES, S. H.; PIMENTA, A. M. C. Síndrome de Burnout em Agentes Comunitários de Saúde e Estratégias de Enfrentamento. Saúde e Sociedade, São Paulo, v. 18, n. 3, p. 467-478, 2009. Disponível em: <http://www.revistas.usp.br/sausoc/article/ view/29616/31484>. Acesso em: 24 nov. 2015.

TRINDADE, L. L. et al. Estresse e síndrome de burnout entre trabalhadores da equipe de Saúde da Família. Acta Paulista de Enfermagem, São Paulo, v. 23, n. 5, p. 684-9, 2010. Disponível em: <http://www.scielo.br/pdf/ ape/v23n5/16.pdf>. Acesso em: 19 jan. 2016.

TUCUNDUVA, L. T. C. M. et al. A síndrome da estafa profissional em médicos cancerologistas brasileiros. Revista da Associação Médica Brasileira, São Paulo, v. 52, n. 2, p. 108-12, 2006.

VIANA, M. T. Trabalhando sem medo: alguns argumentos em defesa da Convenção n. 158 da OIT. Revista do Tribunal Regional dos Trabalhadores da $3^{a}$ Região, Belo Horizonte, v. 46, n. 76, p. 235-246, jul./dez. 2007. Disponível em: <http://www.trt3.jus.br/escola/download/revista/rev_76/Marcio_Viana.pdf>. Acesso em: 2 dez. 2015.

Recebido para publicação em agosto de 2016

Versão final em janeiro de 2017

Conflito de interesses: inexistente

Suporte financeiro: não houve 